\title{
On Detecting Sudden Changes in the Unconditional Volatility of a Time Series
}

\author{
Dilip Kumar \\ Indian Institute of Management, Kashipur, India \\ Email: dilip.kumar@iimkashipur.ac.in,dksic212@gmail.com
}

Received 21 March 2016; accepted 23 April 2016; published 26 April 2016

Copyright (C) 2016 by author and Scientific Research Publishing Inc.

This work is licensed under the Creative Commons Attribution International License (CC BY). http://creativecommons.org/licenses/by/4.0/

c) (i) Open Access

\begin{abstract}
The present study highlights the drawback of using Sanso, Arago and Carrion's (2004) AIT-ICSS algorithm in detecting sudden changes in the unconditional volatility when long memory is present in volatility. Simulation experiments show that the AIT-ICSS test is severely oversized and exhibits low power when long memory is present in volatility.
\end{abstract}

\section{Keywords}

AIT-ICSS Algorithm, Long Memory, Sudden Change, Volatility

\section{Introduction}

Volatility modeling and forecasting play a crucial role in financial market and have been well-researched in the area of economics and finance because of its importance in capital market theories. Volatility of asset returns highlights the risk or uncertainty associated with the asset and, hence, exploring the behavior of volatility of asset returns is relevant for the pricing of financial assets, risk management, portfolio selection, trading strategies and the pricing of derivative instruments [1]. Volatility of asset returns does not remain constant and vary over time; however, sometime we observe sudden changes in volatility of returns. Macroeconomic and political shocks may result in sudden changes in the volatility of asset returns. Such sudden changes in volatility in turn influence the intensity and the direction of information flow across markets, stocks or portfolios as shown by Ross [2]. Hence, detecting sudden changes in the unconditional variance is an important issue in finance, because it is well known that volatility does not stay constant over time. Various approaches are available to detect sudden changes in unconditional volatility. Among them, Inclan and Tiao's [3] Iterated Cumulative Sum of Squares (IT-ICSS) and Sanso, Arago and Carrion's [4] (AIT-ICSS) algorithms are more popular. The AIT-ICSS algorithm deals with the shortcomings of the IT-ICSS algorithm by taking into account conditional heteroskedasticity that is commonly observed in financial time series and also, the serial correlation in the conditional vo- 
latility series. It has met with a great deal of success empirically in being able to identify the breakpoints in volatility regimes [5]-[7].

However, in some instances, it has been applied even when long memory is present in the conditional volatility series, such as by Malik et al. [5], Hammoudeh and Li [6], Wang and Moore [7]. This strikes us as being possibly inappropriate because a careful look at the theory behind the AIT-ICSS algorithm reveals that this procedure is designed to deal only with short term dependence in the conditional volatility series. Hence, it becomes an interesting question to investigate how well the AIT-ICSS algorithm performs under long memory. Therefore, we undertake a simulation experiment to assess the size and power properties of the AIT-ICSS test when long memory is present in volatility using data generating processes from various specifications of the fractionally integrated generalized autoregressive conditional heteroskedasticity (FIGARCH) model. What our results show is that indeed the AIT-ICSS test performs badly under long memory in the sense of being badly oversized. On the application side, we select four major benchmark indices, which are S\&P 500, FTSE 100, Nikkei 225 and CAC 40. We first estimate the fractional integration parameter of these indices using the FIGARCH model and find evidence of long memory in the conditional volatility of these series. We then detect sudden breaks in the unconditional volatility of these series using the AIT-ICSS algorithm and find that most of the detected breaks are spurious in nature, which can be explained based on the evidence from our simulation experiments.

The remainder of this paper is organized as follows: Section 2 introduces the AIT-ICSS algorithm and the associated problem when long memory is incorporated in the series. In Section 3, we undertake Monte Carlo simulation experiments to assess the AIT-ICSS algorithm. Section 4 describes the problem of AIT-ICSS when applied on data and Section 5 concludes with a summary of our main findings.

\section{Methodology}

In the AIT-ICSS test, suppose $\varepsilon_{t} \sim$ iid $\left(0, \sigma^{2}\right)$. Suppose the variance within each interval is given by $\tau_{j}^{2}$, where $j=0,1, \cdots, N_{T}$ and $N_{T}$ is the total number of variance changes in $T$ observations, and $1<k_{1}<k_{2}<\cdots<k_{N T}<T$ are the change points.

$$
\begin{array}{cl}
\sigma_{t}^{2}=\tau_{0}^{2} & \text { for } 1<t<\kappa_{1} \\
\sigma_{t}^{2}=\tau_{1}^{2} & \text { for } \kappa_{1}<t<\kappa_{2} \\
\sigma_{t}^{2}=\tau_{N T}^{2} & \text { for } \kappa_{N T}<t<T
\end{array}
$$

A cumulative sum of squares procedure is used to estimate the number of change points and is given as:

$$
C_{k}=\sum_{t=1}^{k} \varepsilon_{t}^{2}
$$

where $k=1, \cdots, T$. The AIT test statistic to detect sudden change is given as:

$$
A I T=\max _{k} \sqrt{T}\left|G_{k}\right|
$$

where

$$
G_{k}=\frac{1}{\sqrt{\hat{\lambda}}}\left[C_{k}-\left(\frac{k}{T}\right) C_{T}\right], k=1, \cdots, T
$$

where $C_{T}$ is the sum of squared residuals from the whole sample period.

$$
\begin{gathered}
\hat{\lambda}=\hat{\gamma}_{0}+2 \sum_{i=1}^{m}\left[1-\frac{i}{m+1}\right] \hat{\gamma}_{i} \\
\hat{\gamma}_{i}=\frac{1}{T} \sum_{t=i+1}^{T}\left(\varepsilon_{t}^{2}-\hat{\sigma}^{2}\right)\left(\varepsilon_{t-i}^{2}-\hat{\sigma}^{2}\right), \text { and } \hat{\sigma}^{2}=\frac{1}{T} C_{T}
\end{gathered}
$$

It needs to be noted that when we make use of Equation (4.2) we are implicitly assuming that the spectral density at zero of the squared returns is well behaved and, in particular, that as $m$ gets large, the right hand side of Equation (4.2) converges to the following: 


$$
S(0)=\gamma_{0}+2 \sum_{j=1}^{\infty} \gamma_{j}
$$

In practice, the lag truncation parameter $m$ in Equation (4.2) is estimated using the procedure given in Newey and West [8]. The innovation in the AIT-ICSS algorithm with respect to the IT-ICSS algorithm is related to incorporating the correction for conditional heteroskedasticity and also for the possible serial correlation in conditional volatility. However, in Equation (4.2), if $m \rightarrow \infty, \quad \lambda$ will be well behaved only when there is short-term dependence in the conditional volatility series which rules out the presence of long memory in volatility. This is not an ancillary aspect of the AIT-ICSS algorithm, but the essential concept that distinguishes it from the original IT-ICSS algorithm.

\section{Monte Carlo Simulation Experiment}

This section presents the performance of the AIT-ICSS algorithm in the presence of long memory in conditional volatility via simulation. The sample size, the number of Monte Carlo trials and the significance level are taken to be ( $T=100, T=200, T=500$ and $T=1000), 10,000$ and $5 \%{ }^{1}$ respectively. We consider different specifications of FIGARCH $(p, d, q)$ (i.e., FIGARCH $(0, d, 0)$, FIGARCH $(1, d, 0)$ and FIGARCH $(1, d, 1))$ models. The FIGARCH $(p, d, q)$ model is given as:

$$
\varepsilon_{t}=\sqrt{h_{t}} u_{t} ; \phi(L)(1-L)^{d} \varepsilon_{t}^{2}=\omega+[1-\beta(L)] v_{t}, u_{t} \sim \operatorname{iidN}(0,1)
$$

where $v_{t}=\varepsilon_{t}^{2}-h_{t}$ and $\phi(L)=[1-\alpha(L)-\beta(L)]$.

We consider the following cases under (6).

FIGARCH $(1, d, 1)$ :

$$
\left\{\begin{array}{l}
(\omega, \phi, d,(\beta))=(0.1,0.975,0,(0.1,0.3,0.5,0.7)) \\
(\omega, \phi, d,(\beta))=(0.1,0.25,0.5,(0.1,0.3,0.5,0.7)) \\
(\omega, \phi, d,(\beta))=(0.1,0.05,0.75,(0.1,0.3,0.5,0.7))
\end{array}\right.
$$

$\operatorname{FIGARCH}(1, d, 0)$ :

$$
\left\{\begin{array}{l}
(\omega, \phi, d,(\beta))=(0.1,0,0.5,(0.1,0.3,0.45)) \\
(\omega, \phi, d,(\beta))=(0.1,0,0.75,(0.1,0.3,0.5,0.7))
\end{array}\right.
$$

$\operatorname{FIGARCH}(0, d, 0)$

$$
(\omega, \phi,(d), \beta)=(0.1,0,(0,0.25,0.50,0.75), 0)
$$

Under the null hypothesis, there is no sudden change in $\sigma_{t}^{2}$. These specifications can help us to examine the size of the AIT test. For the case of FIGARCH $(1, d, 0)$ model, one specification contains $\beta$ up until 0.45 because for the FIGARCH model, $(\phi+d-\beta)$ should be non-negative. Under the alternative hypothesis, there exists a sudden change in volatility; hence, we incorporate a break at the 50th percentile ${ }^{2}$ of the series to compute the power of the test. To generate $\varepsilon_{t}^{2}$ for assessing the power of the test, we first generate $\varepsilon_{t}$ using the respective FIGARCH specification and keep first part of the series as it is and multiply the second half by $(1+\lambda)$, where $\lambda$ indicates the percentage change in the unconditional volatility of the series. Here the results for FIGARCH $(1, d, 1)$ and FIGARCH $(1, d, 0)$ specifications are very similar and due to lack of space we only report the results of all FIGARCH $(1, d, 1)$ specifications. We also report the power of the test for FIGARCH $(0, d, 0)$ for different values of $d(0,0.5$ and 0.75$)$.

Table 1 presents the results of size of the test for different specifications of the FIGARCH $(p, d, q)$ model. The results indicate that the AIT-ICSS test is severely oversized for all the cases with the exception of FIGARCH $(0, d, 0)$ model and FIGARCH $(1, d, 1)$ model (for $\beta=0.10)$ when $d=0$.

Table 2 reports the power of the test. The FIGARCH $(0, d, 0)$ model with $d=0$ exhibits good power for a

\footnotetext{
${ }^{1}$ We also perform simulation experiments by setting significance level at $1 \%$ as well as $10 \%$ and the results (not shown here and available on request) give rise to a similar inference as obtained in these simulations.

${ }^{2}$ We also perform the same analysis by incorporating breaks at $25^{\text {th }}$ percentile as well as $75^{\text {th }}$ percentile and obtain similar results. (The results are not shown here and will be available on request).
} 
Table 1. Size of the test.

\begin{tabular}{|c|c|c|c|c|c|c|c|c|c|}
\hline & \multicolumn{4}{|c|}{$\operatorname{FIGARCH}(1, d, 1)(d=0 \& \phi=0.975)$} & \multicolumn{5}{|c|}{ FIGARCH $(0, d, 0)$ for different values of $d$} \\
\hline$\beta$ & $T=100$ & $T=200$ & $T=500$ & $T=1000$ & $d$ & $T=100$ & $T=200$ & $T=500$ & $T=1000$ \\
\hline 0.10 & 0.044 & 0.042 & 0.052 & 0.055 & 0.00 & 0.017 & 0.024 & 0.033 & 0.035 \\
\hline 0.30 & 0.095 & 0.088 & 0.113 & 0.115 & 0.25 & 0.106 & 0.179 & 0.332 & 0.464 \\
\hline 0.50 & 0.191 & 0.202 & 0.266 & 0.263 & 0.50 & 0.149 & 0.256 & 0.484 & 0.629 \\
\hline \multirow[t]{2}{*}{0.70} & 0.275 & 0.403 & 0.489 & 0.541 & 0.75 & 0.103 & 0.170 & 0.253 & 0.303 \\
\hline & \multicolumn{4}{|c|}{$\operatorname{FIGARCH}(1, d, 1)(d=0.5 \& \phi=0.25)$} & \multicolumn{5}{|c|}{$\operatorname{FIGARCH}(1, d, 0)(d=0.5 \& \phi=0)$} \\
\hline$\beta$ & $T=100$ & $T=200$ & $T=500$ & $T=1000$ & $\beta$ & $T=100$ & $T=200$ & $T=500$ & $T=1000$ \\
\hline 0.10 & 0.129 & 0.214 & 0.366 & 0.520 & 0.10 & 0.179 & 0.299 & 0.491 & 0.652 \\
\hline 0.30 & 0.145 & 0.311 & 0.484 & 0.646 & 0.30 & 0.213 & 0.391 & 0.621 & 0.756 \\
\hline 0.50 & 0.190 & 0.365 & 0.615 & 0.753 & 0.45 & 0.235 & 0.407 & 0.649 & 0.806 \\
\hline \multirow[t]{2}{*}{0.70} & 0.156 & 0.366 & 0.649 & 0.793 & - & - & - & - & - \\
\hline & \multicolumn{4}{|c|}{$\operatorname{FIGARCH}(1, d, 1)(d=0.75 \& \phi=0.05)$} & \multicolumn{5}{|c|}{$\operatorname{FIGARCH}(1, d, 0)(d=0.75 \& \phi=0)$} \\
\hline$\beta$ & $T=100$ & $T=200$ & $T=500$ & $T=1000$ & $\beta$ & $T=100$ & $T=200$ & $T=500$ & $T=1000$ \\
\hline 0.10 & 0.129 & 0.188 & 0.298 & 0.349 & 0.10 & 0.151 & 0.192 & 0.331 & 0.349 \\
\hline 0.30 & 0.193 & 0.293 & 0.436 & 0.498 & 0.30 & 0.192 & 0.286 & 0.443 & 0.533 \\
\hline 0.50 & 0.275 & 0.422 & 0.616 & 0.700 & 0.50 & 0.294 & 0.441 & 0.657 & 0.744 \\
\hline 0.70 & 0.326 & 0.563 & 0.773 & 0.890 & 0.70 & 0.319 & 0.525 & 0.784 & 0.914 \\
\hline
\end{tabular}

Table 2. Power of the test.

\begin{tabular}{|c|c|c|c|c|c|c|c|c|}
\hline \multirow[b]{2}{*}{ Lambda } & \multicolumn{4}{|c|}{ FIGARCH $(1, d, 1)$ with $d=0 \& \phi=0.975$} & \multicolumn{4}{|c|}{ FIGARCH $(0, d, 0)$ with $d=0$} \\
\hline & $T=100$ & $T=200$ & $T=500$ & $T=1000$ & $T=100$ & $T=200$ & $T=500$ & $T=1000$ \\
\hline 0.10 & 0.301 & 0.412 & 0.515 & 0.526 & 0.036 & 0.064 & 0.206 & 0.415 \\
\hline 0.30 & 0.346 & 0.466 & 0.583 & 0.615 & 0.170 & 0.482 & 0.939 & 1.000 \\
\hline 0.50 & 0.373 & 0.502 & 0.665 & 0.732 & 0.413 & 0.880 & 0.999 & 1.000 \\
\hline 1.00 & 0.479 & 0.681 & 0.826 & 0.900 & 0.857 & 1.000 & 1.000 & 1.000 \\
\hline \multirow[t]{2}{*}{3.00} & 0.774 & 0.887 & 0.943 & 0.976 & 0.996 & 1.000 & 1.000 & 1.000 \\
\hline & \multicolumn{4}{|c|}{ FIGARCH $(1, d, 1)$ with $d=0.5 \& \phi=0.25$} & \multicolumn{4}{|c|}{ FIGARCH $(0, d, 0)$ with $d=0.5$} \\
\hline Lambda & $T=100$ & $T=200$ & $T=500$ & $T=1000$ & $T=100$ & $T=200$ & $T=500$ & $T=1000$ \\
\hline 0.10 & 0.194 & 0.400 & 0.631 & 0.798 & 0.171 & 0.274 & 0.462 & 0.600 \\
\hline 0.30 & 0.290 & 0.497 & 0.759 & 0.854 & 0.161 & 0.278 & 0.517 & 0.625 \\
\hline 0.50 & 0.467 & 0.713 & 0.859 & 0.919 & 0.209 & 0.397 & 0.607 & 0.745 \\
\hline 1.00 & 0.773 & 0.921 & 0.984 & 0.998 & 0.374 & 0.593 & 0.783 & 0.875 \\
\hline \multirow[t]{2}{*}{3.00} & 0.984 & 0.999 & 1.000 & 1.000 & 0.693 & 0.895 & 0.976 & 0.989 \\
\hline & \multicolumn{4}{|c|}{ FIGARCH $(1, d, 1)$ with $d=0.75 \& \phi=0.05$} & \multicolumn{4}{|c|}{ FIGARCH $(0, d, 0)$ with $d=0.75$} \\
\hline Lambda & $T=100$ & $T=200$ & $T=500$ & $T=1000$ & $T=100$ & $T=200$ & $T=500$ & $T=1000$ \\
\hline 0.10 & 0.336 & 0.522 & 0.765 & 0.900 & 0.109 & 0.168 & 0.259 & 0.303 \\
\hline 0.30 & 0.371 & 0.547 & 0.783 & 0.884 & 0.111 & 0.157 & 0.283 & 0.382 \\
\hline 0.50 & 0.458 & 0.636 & 0.812 & 0.914 & 0.142 & 0.220 & 0.372 & 0.465 \\
\hline 1.00 & 0.634 & 0.788 & 0.894 & 0.937 & 0.219 & 0.367 & 0.547 & 0.635 \\
\hline 3.00 & 0.925 & 0.984 & 0.992 & 0.997 & 0.451 & 0.645 & 0.824 & 0.868 \\
\hline
\end{tabular}


sudden change in the unconditional volatility of $100 \%$ or more and the power of the test increases as sample size increases even for sudden change of $30 \%$ or more. On the other hand, for non-zero $d$, we observe good power for other specifications only when there is sudden change of $300 \%$ or more for sample size greater than 200 . The FIGARCH $(0,0.75,0)$ model exhibits the worst power of all the specifications under study.

This indicates that when long memory is present in the conditional volatility of a time series, the AIT-ICSS algorithm breaks down and is no longer suitable for detecting sudden changes in its unconditional volatility.

\section{Application}

We apply the AIT-ICSS algorithm on weekly data of four major benchmark series having long memory in the conditional volatility. The data period is from January 1996 to March 2015. Table 3 reports the estimated values of long memory parameter $\mathrm{d}$ using the FIGARCH $(1, d, 1)$ specification. The results clearly show that there exists long memory in the conditional volatility of all the series under study.

Table 4 reports the breaks detected using the AIT-ICSS algorithm for the given four series. We detect four break points in the S\&P 500, three break points in FTSE 100, zero break points in Nikkei 225 and four break points in CAC 40 which represent the presence of $(n+1)$ distinct volatility regimes in the time series. It can be seen that most of the detected breaks cannot be related to major macroeconomic or political events and, hence, are probably spurious. This may well be due to the drawback associated with the AIT-ICSS algorithm that the test is severely oversized when long memory is present in the volatility of the series. Our findings are similar to what was found by Schwert [9] in that it is difficult to explain sudden changes in volatility with corresponding macroeconomic events.

Table 3. Estimates of $d$ in the FIGARCH model.

\begin{tabular}{ccccc}
\hline & S\&P_500 & FTSE_100 & Nikkei_225 & CAC_40 \\
\hline$d$ & $0.527^{\#}$ & $0.512^{\#}$ & $0.424^{\#}$ & $0.588^{\#}$ \\
SE & $(0.162)$ & $(0.188)$ & $(0.142)$ & $(0.182)$ \\
\hline
\end{tabular}

\# and ${ }^{*}$ mean significant at $1 \%$ and $5 \%$ level of significance. SE represents the standard error.

Table 4. Breaks detected.

\begin{tabular}{|c|c|c|c|}
\hline Index (period) & $\begin{array}{c}\text { Number of change } \\
\text { points }\end{array}$ & Time period & Events \\
\hline \multirow{5}{*}{ S\&P_500 } & \multirow{5}{*}{4} & 05-05-1982 to 03-08-1988 & - \\
\hline & & $10-08-1988$ to $22-04-1992$ & - \\
\hline & & 29-04-1992 to $15-07-1998$ & - \\
\hline & & $22-07-1998$ to $11-07-2007$ & Russian financial crisis \\
\hline & & $18-07-2007$ to $28-11-2012$ & Global financial crisis \\
\hline \multirow{4}{*}{ FTSE_100 } & \multirow{4}{*}{3} & 04-01-1984 to 08-07-1998 & - \\
\hline & & $15-07-1998$ to $16-03-2003$ & - \\
\hline & & $23-03-2003$ to $17-10-2007$ & - \\
\hline & & $24-10-2007$ to $28-11-2012$ & Global financial crisis \\
\hline Nikkei_225 & 0 & - & No break detected \\
\hline \multirow{5}{*}{ CAC_40 } & \multirow{5}{*}{4} & 06-01-1988 to 05-08-1998 & - \\
\hline & & $12-08-1998$ to $10-07-2002$ & Russian financial crisis \\
\hline & & $17-07-2002$ to $19-03-2003$ & - \\
\hline & & $16-03-2003$ to $09-01-2008$ & - \\
\hline & & $16-01-2008$ to $28-11-2012$ & Global financial crisis \\
\hline
\end{tabular}




\section{Conclusion}

Our simulation experiment indicates that the AIT-ICSS test is severely oversized and exhibits low power when long memory is present in the volatility of a time series. The empirical analysis also indicates that most of the breaks detected by the AIT-ICSS test cannot be related to major macroeconomic and political events and, hence, are probably spurious.

\section{References}

[1] Poon, S.H. and Granger, C.W.J. (2003) Forecasting Volatility in Financial Markets: A Review. Journal of Economic Literature, 41, 478-539. http://dx.doi.org/10.1257/.41.2.478

[2] Ross, S.A. (1989) Information and Volatility: The No-Arbitrage Martingale Approach to Timing and Resolution Irrelevancy. Journal of Finance, 44, 1-7. http://dx.doi.org/10.1111/j.1540-6261.1989.tb02401.x

[3] Inclán, C. and Tiao, G.C. (1994) Use of Cumulative Sums of Squares for Retrospective Detection of Changes of Variance. Journal of the American Statistical Association, 89, 913-923.

[4] Sanso, A., Arago, V. and Carrion, J.L. (2004) Testing for Change in the Unconditional Variance of Financial Time Series. Revista de Economiá Financiera, 4, 32-53.

[5] Malik, F., Ewing, B.T. and Payne, J.E. (2005) Measuring Volatility Persistence in the Presence of Sudden Changes in the Variance of Canadian Stock Returns. Canadian Journal of Economics, 38, 1037-1056. http://dx.doi.org/10.1111/j.0008-4085.2005.00315.x

[6] Hammoudeh, S. and Li, H. (2008) Sudden Changes in Volatility in Emerging Markets: The Case of Gulf Arab Stock Markets. International Review of Financial Analysis, 17, 47-63. http://dx.doi.org/10.1016/j.irfa.2005.01.002

[7] Wang, P. and Moore, T. (2009) Sudden Changes in Volatility: The Case of Five Central European Stock Markets. Journal of International Financial Markets, Institutions and Money, 19, 33-46. http://dx.doi.org/10.1016/j.intfin.2007.08.006

[8] Newey, W.K. and West, K.D. (1994) Automatic Lag Selection in Covariance Matrix Estimation. Review of Economic Studies, 61, 631-654. http://dx.doi.org/10.2307/2297912

[9] Schwert, G.W. (1989) Why Does Stock Market Volatility Change over Time? The Journal of Finance, 44, 1115-1153. http://dx.doi.org/10.1111/j.1540-6261.1989.tb02647.x 